\title{
On the Development of Multi Agent System for Large Urban Area Evacuation with Autonomous Navigation
}

\author{
Maddegedara Lalith*, Leonel A. Melgar**, and Muneo Hori*** Tsuyoshi Ichimura*** \\ Seizo Tanaka ${ }^{\dagger}$ \\ ${ }^{*}$ Associate Professor, Earthquake Research Institute, University of Tokyo (Bunkyo, Tokyo 113-0032) \\ E-mail: lalith@eri.u-tokyo.ac.jp \\ ${ }^{* *}$ Graduate Students, Department of Civil Engineering, University of Tokyo (Bunkyo, Tokyo 113-8656) \\ E-mail:leaguilar@uvg.edu.gt \\ ${ }^{* * *}$ Professor, Earthquake Research Institute, University of Tokyo (Bunkyo, Tokyo 113-0032) \\ E-mail: hori@eri.u-tokyo.ac.jp \\ ${ }^{* * * *}$ Accos. Professor, Earthquake Research Institute, University of Tokyo (Bunkyo, Tokyo 113-0032) \\ E-mail: ichimura@eri.u-tokyo.ac.jp \\ ${ }^{\dagger}$ Asst. Professor, Earthquake Research Institute, University of Tokyo (Bunkyo, Tokyo 113-0032) \\ E-mail: stanaka@eri.u-tokyo.ac.jp
}

\begin{abstract}
With the aim of estimating the effectiveness of the measures taken to smoothen and speedup the evacuation process of a large urban area, in time critical events like tsunami, a multi agent based mass evacuation simulation software is being developed. Considering the fact that it involves large number of human casualties, moderately complex agents in 2D grid environments are implemented. A vision based autonomous navigation algorithm, which enables the agents to move through an urban environment and reach a far visible destination, is implemented. The navigation algorithm is verified comparing the simulated evacuation time and the paths taken by individual agents with those of theoretical. We adopted optimal reciprocal collision avoidance algorithm for collision avoidance and validated by comparing with filed observations reported in literature. A parallel computing extension is developed for studying mass evacuation of large areas; simulating millions of agents with vision based navigation and collision avoidance is computationally intensive. A high strong scalability is attained with two millions of agents, which indicates potential to simulate mass evacuation involving many millions of people.
\end{abstract}

Key Words : mass evacuation; multi agent systems; autonomous navigation; parallel computing

\section{Introduction}

Large number of casualties due to natural disasters like the two tsunamis in Sumatra and Japan emphasize the need of understanding the dynamics of mass evacuation in earthquake engineering. Such studies will help to identify possible bottlenecks, find strategies to make the evacuation process smooth and reduce evacuation time, etc., eventually saving many human lives. A major barrier in mass evacuation studies is the inability to conduct experiments; small scale experiments do not give a correct picture of large scale problem, and experiments are often dangerous and difficult to conduct. There are several numerical methods being used for evacuation simulations, like cellular automata, social force model, etc. Out of these Multi Agent System (MAS) is a promising method for modeling heterogeneous and complex human behavior involved in mass evacuations 1),2),3),4),5). Most of the existing studies of tsunami triggered mass evacuation with MAS use simpli- fied environment with simple agents ${ }^{1), 2), 5)}$. Often the environment is modeled as a network consisting of $1 \mathrm{D}$ members which represent rooms, corridors, roads, open spaces, etc., each associated with a pedestrian capacity, while the agents have basic properties like velocity. Most common objective is to identify the possible problems when agents try to reach a predefined shelter along a predefined path.

Considering the fact that tsunami evacuation is directly concerned with tens to hundreds of thousands of human lives, more sophisticated modeling is necessary. People recognize problems like congestions, blocked roads and find new paths to reach a safe destination according to the risks they perceive. Further, they help others and seek for the information they require. It is necessary to consider such behaviors which have significant effects and use a sufficiently detailed model of environment to support complex agents' behaviors. Especially, such complex MAS models are essential for real-time monitoring, predicting and guiding systems, which is realizable within 
next decades. Such system will: monitor each individual via mobile networks; forecast crowd behavior, considering various possibilities (i.e. Monte-Carlo simulations); choose the best possible routes for each individual based on the predictions; actively control the crowd by sending the instructions to reach a safe place avoiding congestions, damaged roads and hazards like fire. In addition, the system must analyze the human behavior to identify any blocked routes and take those into the account when predicting; changes to the environment is unknown right after an earthquake.

Working towards above mentioned real-time monitoring, predicting and guiding system, we implemented a moderately complex MAS with autonomously navigating agents in 2D urban environments, for large area evacuation simulation. The main input for agents' autonomous navigation is the details of their visible neighborhood which they perceive with their sense of vision and hearing. Collision avoidance is another essential ingredient in evacuation simulations and a sophisticated algorithm which can reproduce the filed observations has to be implemented. We adopted optimal reciprocal collision avoidance algorithm ${ }^{6}$ and validated by comparing with filed observations. Simulating millions of agents with vision based autonomous navigation and collision avoidance is computationally intensive. To meet this high computational demand, we developed a scalable parallel extension with the Message Passing Interface (MPI) ${ }^{7}$. Our short term target of this system is to, for a given environment, test the effectiveness of different strategies for smooth and fast evacuation.

The contents of the rest of this paper are organized as follows. Section two gives a brief introduction to the developed MAS system for mass evacuation simulations. Details of the vision based autonomous navigation and collision avoidance are given in the section three. Verification of autonomous navigation algorithm and validation of collision avoidance are given in the fourth section. The section five briefly presents the implemented parallel computing strategies. Finally, some concluding remarks are presented in the last section.

\section{A multi agent system for evacuation sim- ulations}

The main two elements of our multi agent system are agents and environment. The agents are the software counterparts which mimic the relevant behavior of people in a mass evacuation event. Agents interact with each other and the environment taking spontaneous actions. The rest of this section gives a brief overview of these two elements, focusing on the basic requirements of these two elements and implementations.

\subsection{Environment}

The environment is modeled as a high resolution grid, with the maximum grid interval of $1 \mathrm{~m}$. The choice of grid model for the environment, instead of the widely used vector model, is due to three main reasons; ease of modifying the environment, identifying boundary of visibility and parallel computing.

Vector models are widely used due to their versatility and compactness. Elements like buildings, roads, etc. are modeled as closed polygons and detailed information like number of lanes, types of the building, number of occupants, etc. are embedded into these polygons. With vector models, route planning is fast and smooth edges of elements make the agent navigation easier. On the other hand, the grid model does not have any of these advantages: buildings, roads, etc. are no longer identifiable single entities; route planning in grid environment is time consuming; agent navigation in saw-toothed grid environment poses difficulties.

However, some advantages of grid environment make it a good candidate, provided the problem of route planning can be solved. Tsunami triggered evacuation requires real-time modification of the environment to incorporate the earthquake induced blockage of roads. Such localized modifications can be efficiently made in grid environments. Also, identifying an agent's visible horizon is simple and fast in grid environments; working with concave polygons of vector models is complex. Further, the vector model poses several difficulties in parallel computing; domain partitioning, dynamic load balancing and communicating graphs of vector data are complex, and have lower scalability compared to the grid data. When the region being modeled is several tens or hundreds of square kilometers, grid environment is much simpler to manage in parallel computing. The choice of grid environment is due to these advantages; how to accelerate route panning in grid environment is explained in the latter part of this section.

\subsection{Agents}

An agent has two sets of data pertaining to its inherent properties, like maximum speed, age, maximum sight distance, etc., and its memory holding the information like past experiences, the routes it has taken, destination, etc. An agent has several major functionalities; think, see and move. To mimic real heterogeneous crowds, agents with wide range of physical abilities are generated according to a given distribution. Further, the agents are specialized by including more information and functionalities to represent relevant officials like police officers, fire fighters, volunteers, etc.

While we can include many different types of agents, relevant to the topic of autonomous navigation are two categories of agents; visitors and non-visitors. A visitor agent does not possess any information about the environment, except what is to be seen within its visible horizons, what is learnt while moving around, and the direction to a far visible destination like high ground, if any. All except vis- 
itor agents (e.g. residents, police officers, etc.) have the information of their neighborhood and have the ability to find the route to a desired destination. Here onwards, all types of agents except the visitor agents are called nonvisitor agents.

\section{(1) Radar vision}

Vision is the main mean of perceiving the environment for human beings. Just as the human counterpart, agents must be able to extract information within a neighborhood of several tens of meters of his sight distance and identify the features of the environment; features like buildings, roads, etc. are not directly recognizable in an environment modeled as a grid. We implemented radar based vision to make the agents identify the features like roads, obstacles, etc., and neighboring agents, within their sight distances.

The vision functionality scans the environment like radar and identifies the boundary of an agent's visibility, in high resolution. The resolution of radar scanning, $d \theta$, is set such that $L \times d \theta=0.5 \times \delta l$, where $L$ is the sight distance and $\delta l$ is the length of grid cell; approximately 1000 rays for $80 m$ sight distance. Fig. 1 shows the identified boundary of visibility and neighbor agents, by an agent with $40 \mathrm{~m}$ sight distance. Analyzing the boundary of visibility an agent identifies the available paths and moves towards the chosen direction, avoiding collision.

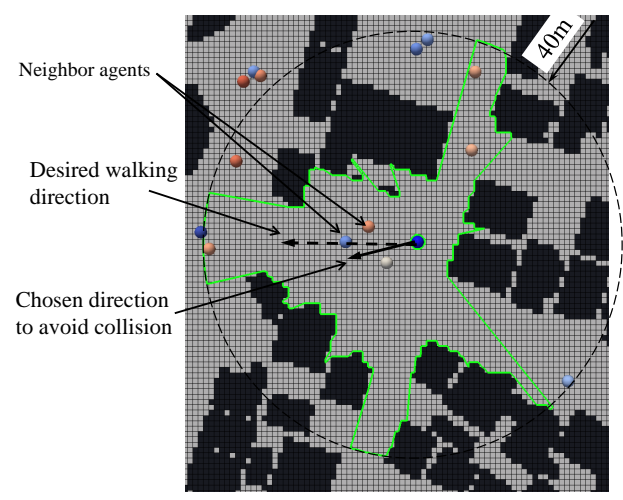

Fig. 1: Shown in green is the boundary of visibility.

Radar vision is computationally intensive since it requires ray tracing. In grid environments, floating point operations involved in ray tracing can be significantly reduced by using pre-calculated templates. For the current study, 16 sets of radar templates with their origins arranged in a $4 \times 4$ grid of $20 \mathrm{~cm}$ spacing are used. Each radar templates contains the information of cells through which rays pass and the intersecting points. An agent chooses the best radar template according to their location in a grid cell of $1 m \times 1 m$.

\subsection{Route planning}

Real-time route finding is an essential for the target problem; if an agent finds the route its following is blocked by a collapsed building, etc., it should be able to find the next closest route to a nearby high ground or evacuation center. A major problem with the grid environments is

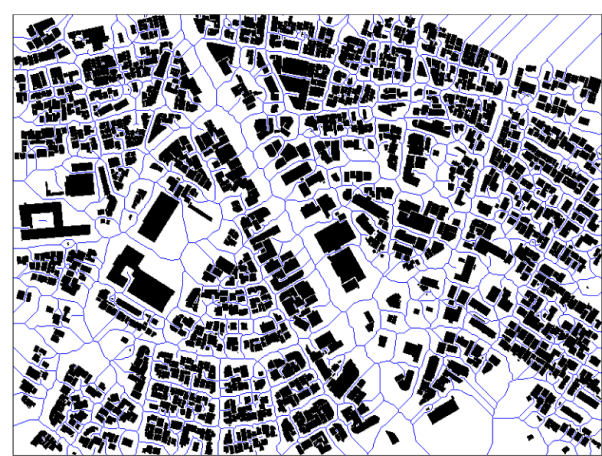

(a) Pixel skeleton obtained by thinning.

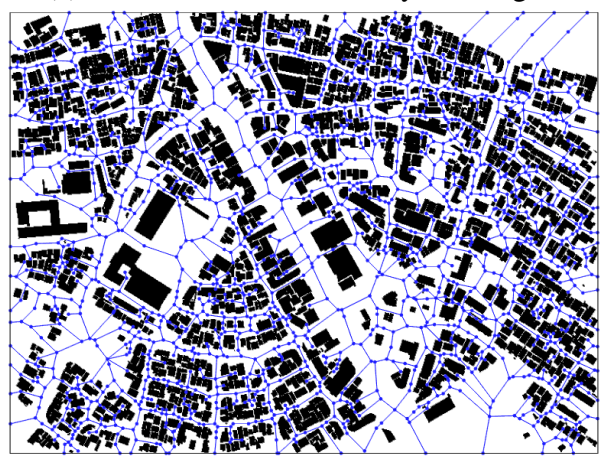

(b) Vector map of available routes.

Fig. 2: Automated route map generation from grid data. Blue dots in (b) are the nodes defining the vector map.

the complexity of route planning algorithms. Real-time route finding in large grids is extremely slow and drastically reduces parallel scalability. This problem can be solved by using a vector map for route planning. Vector map of available routes in a grid model can be automatically constructed by abstracting the pixel skeletons obtained with thinning algorithms ${ }^{8}$. Fig. $2 \mathrm{a}$ and $2 \mathrm{~b}$ show a pixel skeleton obtained with thinning algorithms and the corresponding vector map, respectively.

\section{Autonomous navigation in urban envi- ronments and collision avoidance}

For the target problem of tsunami induced large area evacuations, the agents must have good autonomous navigation skills to navigate through a complex urban environment which is modeled as a grid. At least the agents should have the ability of navigating to a far visible high ground or a tall building, even if an agent represents a visitor; an essential skill which any human counterpart possesses. Also, collision avoidance is an essential item for modelling change of walking velocities with the density of crowd, which accounts for increase of evacuation time with crowd density. This section provides the details of the autonomous navigation algorithm and collision avoidance. These algorithms use the radar vision explained in the previous section, to identify the features of the visible environment. Verification of autonomous navigation and validation of collision avoidance are presented in next sec- 
tion.

\subsection{Identification of features of the visible horizon}

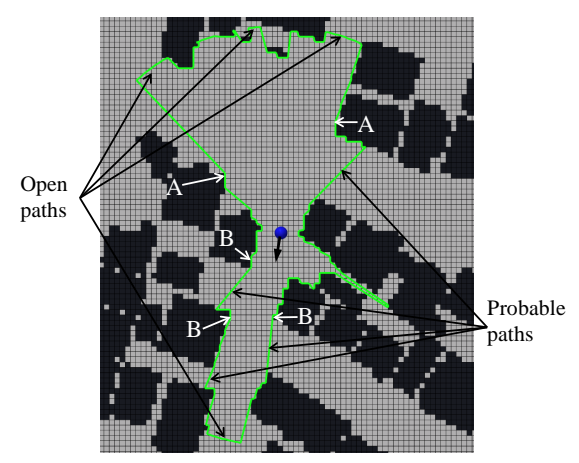

(a) Available open and probable paths.

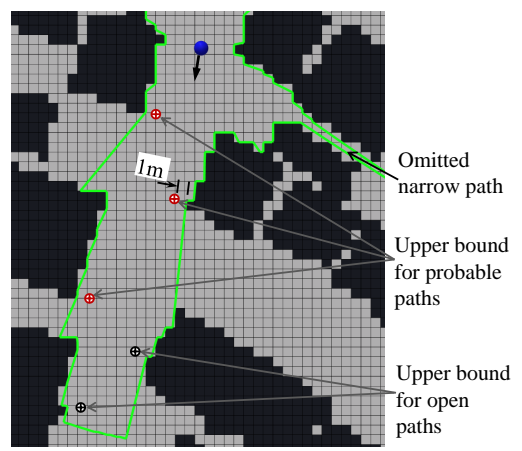

(b) Target points for preventing obstruction of vision.

Fig. 3: Identification of available paths for the agent shown in blue.

For autonomous navigation, an agent must be able to identify all the available paths to take and choose a proper direction to go. Analyzing the boundary of visibility, agents identify two types of paths; open-paths and probable-paths. The regions visible up to an agents sight distance are categorized as open-paths, while the sudden jumps greater than a selected value are categorized as probable-paths (see Fig. 3a).

Correct identification of probable-paths is necessary to make an agent follow a road with sharp bends or junctions. Some care has to be taken when identifying the available probable-paths since, in grid environments, saw-toothed boundary of visibility often produce sudden jumps similar to those produce by roads at a junction. Moving towards these false signals not only produces longer evacuation time compared to that of the human counterparts, but also complex coding is required to bring the agents back to their original route. In order to eliminate these false signals, only the jumps greater than $5 m$ are considered.

Care has to be taken not to let an agent to move too close to any corner which gives rise to sudden jumps (e.g. points shown with A and B in Fig. 3a). Moving too close to a corner of an obstacle significantly lowers an agent's field of vision, often forcing it to turn backwards. In order to prevent this problem, associated with each open or probable paths are a set of points defining the closest they can move towards the corresponding corners of obstacles. Fig. $3 b$ shows some of the points defining the closest an agent can move to corners giving rise to sudden jumps in boundary of visibility. It is found that setting these upper bound points at $1 \mathrm{~m}$ away from the obstacle corners solves the problem of view obstruction.

\subsection{Navigation of non-visitor agents}

Non-visitor agents have the ability to plan a route to a desired destination and their paths are defined by a few number of landmark points, which could be several hundred meters apart. To move between the landmark points defining their route, non-visitor agents use vision based navigation. The selection of proper direction to move is rather simple for non-visitor agents. Being aware of the environment, non-visitor agents considers all the available open or probable paths in his front and back with equal priority, in choosing the closest path towards the next landmark point of its pre-planned route.

\subsection{Navigation of visitor agents}

Unlike the non-visitors, the visitor agents neither have the ability to find paths nor a well-defined destination. In general, visitor agents have several methods to reach a safe destination; get information from non-visitor agent or mass media, follow a group or a leader, etc. However, in the case of tsunami evacuation, safe locations like far visible high ground or tall buildings could be abundant. Human counterparts can navigate to such a far visible destination through unknown urban environments. Hence the visitor agents require the ability to autonomously navigate to a far visible safe location all by themselves.

Making an agent autonomously navigate through an unknown urban environment only using the information in few tens of meters visibility, is not trivial. It is found that choosing open and probable paths according to the priority given in the Algorithm 1 makes a visitor agent to navigate through a urban environment and reach its destination. Also, it is found that setting the sight distance almost equal to the distance between two lanes in the considered urban environment produces the best results. With the problem considered in the next section, sight distance of $40 \mathrm{~m}$ to $50 \mathrm{~m}$ (see Fig. 4) made most of the visitor agents navigate to their destination. Though it is not perfect, Algorithm 1 produces fairly good results as shown in the next section.

Once the open or probable path to be taken is decided, an agent checks for possible collisions with neighboring agents and slightly adjusts the moving direction to avoid collision (see Fig. 1), sticking to the selected open or probable path. 


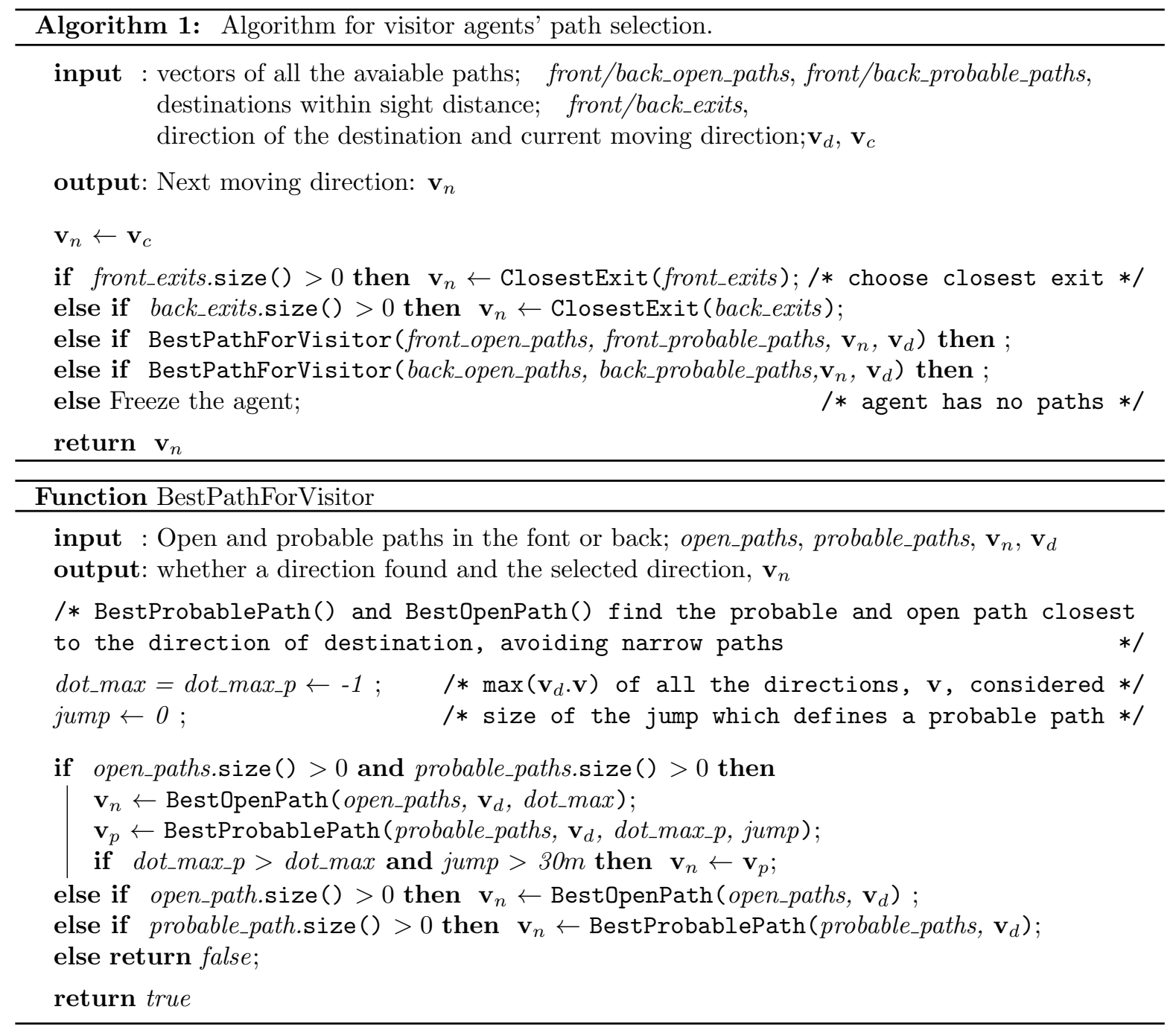

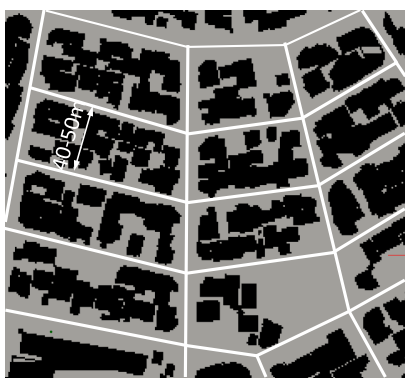

Fig. 4: Distance between parallel lanes in a residential area is found to be a good choice for the sight distance of visitor agents.

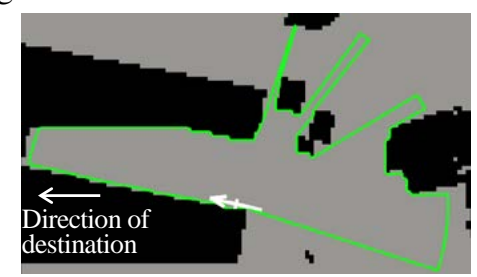

Fig. 5: An agent moving to a well deeper than it's sight distance may have a low chance of getting out of it, depending on the arrangement of the paths avaiable at the entrance.

\section{(1) Known limitations of visitor agent's navigation}

There is one known limitation of visitor agents' navigation; non-visitors have no known limitations. A visitor agent might get lost and start to move in a loop, if it enters a region where there are no identifiable opening towards the destination, and depth of the region longer than the sight distance. Under such circumstances Algorithm 1 makes the agent to turn back and exit such deep wells. However, some complex setting at the mouth of the deep well may make the agent re-enter the deep well, making it move in a loop eternally. Fig. 5 shows an agent entering such a region. When the agent recognizes there is no path towards its destination, it turns and starts moving out of the deep well. However the upward path at the entrance to the deep well makes it turn back and re-enter the deep well, eternally making the agent to move in a closed loop. A simple solution to this problem is temporarily increasing such trapped agents' sight distance to prevent reentering the deep well.

\subsection{Collision avoidance}

Collision avoidance is another essential feature for evacuation simulations. The collision avoidance should be 
sophisticated enough to reproduce observed changes of pedestrian velocities with the density of the crowd, in addition successfully avoiding collision with similar walking behaviors observed. We adopted Optimal Reciprocal Collision Avoidance (ORCA) algorithm ${ }^{6}$, out of many other available techniques like social force model ${ }^{9}$ ), reciprocal velocity objects $^{10)}$, etc.

For the sake of completeness, the ORCA algorithm is briefly explained here and interested readers are referred to the original work. The optimal collision avoidance scheme identifies the set of all relative velocities that would bring two agents to a collision state, which is called a velocity object, finds the smallest change in the relative velocity that would bring two agents to the critical velocity that would evade the collision, and creates a line through that point. This line tangent to the velocity object separates the velocity domain into two half planes. The half plane containing the collision free velocities is what is called an ORCA-plane. In order to avoid collision among several agents, these ORCA-planes are generated for each of the neighbors of an agent and the optimal for this agent solution is found by linear programing.

When implementing ORCA algorithm in vision based navigation, two ORCA planes are generated connecting the agent's position and the two limiting points associated with the opening selected by the agent's navigation algorithm; as explained in the section 3.1, these points define the closest an agent can move towards corners of obstacles. These ORCA planes along with the neighbors' ORCA planes are considered in a certain order of priority; navigation ORCA planes have the highest priority followed by the ORCA planes of neighbors according to the ascending distance. This allows us to loosen constrains by eliminating ORCA planes with less priority, when a collision free state is not found.

\section{Verification and validation of au- tonomous navigation and collision avoidance}

Verification and validation of the proposed radar vision based autonomous navigation and collision avodance are essential since the simulated process are people in an evacuation scenario, with the aim of reducing casualties. For verification, it has to be tested whether the proposed algorithm can reproduce the analytically expected answer for a well-posed problem. Validation requires testing whether the numerically obtained solution satisfactorily matches with the observed behavior of a real evacuation.

For the autonomous navigation, we consider verification by comparing the overall and agent-wise results with analytically obtained shortest paths and evacuation times. The problem for the verification is chosen so as to evaluate the proposed algorithms in navigating agents to far visible destinations. For the collision avoidance, validation is performed by comparing with real observations reported in literature.

\subsection{Verification of navigation algorithm}

For the verification tests, 4000 agents in $1.6 \times 1.2 \mathrm{~km}^{2}$ regions of Kochi city environment is considered; as shown in Fig. 8 the selected area has many buildings and a few open areas. Two cases, each with either non-visitor agents or visitor agents, are considered. The agents are expected to move to a high ground, which is visible from anywhere in the selected domain, located at the left edge of the domain. All the agents are assumed to have the same sight distance of $50 \mathrm{~m}$. The non-visitor agents follow the shortest routes to the left edge of the boundary from their starting location. On the other hand, visitor agents navigate only based on the information within their sight distance and the direction to the visible high ground. Apart from these, both of the problems have the same settings.

The collision avoidance or other interactions among agents are not considered in the verification of navigation since navigation and collision avoidance are independent. Surely, it is important to study to which extent the agents' behavior deviates when the collision avoidance is included and how it varies with the crowd density. However, it is out of the scope of the current study.

\section{(1) Comparison with the ideally expected time}

Fig. 6 shows the graphs of cumulative number of agents exited versus time. The expected ideal number of egressed agents was calculated by finding each agent's individual optimal path to the nearest evacuation point using Theta* algorithm $^{11)}$ and calculating the time it would take them to follow that path without any deviation. The near perfect matching of non-visitor graph with that of the expected ideal verifies non-visitor agents' navigation capabilities; even if they rely on their vision to navigate between few landmark points defining their routes, they can reach the destinations at the ideally expected time. As seen in the Fig. 6, cumulative graph of visitor agents is close the expected and have the maximum difference of 260 agents at 1600 seconds. Further, Fig. 7 shows the distribution of the difference between visitor agents' actual evacution time and expected ideal evacuation time. As is seen, only a few number of agents have more than 4 minutes delay compared to the ideal time and the number of agents with greater than 5 minutes delay is neglegible. The authors think such deviation from the ideal is acceptable considering the complexity and size of the domain.

\section{(2) Trajectories of individual agents}

In addition to the overall behavior, investigating the paths taken by each individual is important to make sure that none of the agents has taken paths which are unrealistic choices for the human counterpart. The possibility of non-visitor agents to take an unrealistic path is very low since they are guided with intermediate landmark points. Analysis of simulation results confirmed that all the non-visitor agents have followed the pre-planned shortest routes with minor deviations.

As one can easily guess, visitor agents have the highest 


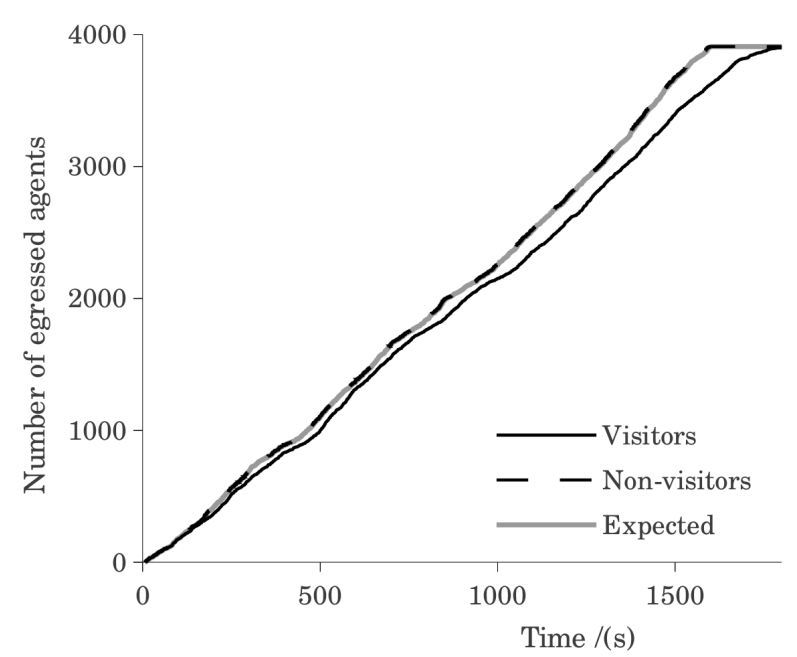

Fig. 6: Cumulative number of agents exited.

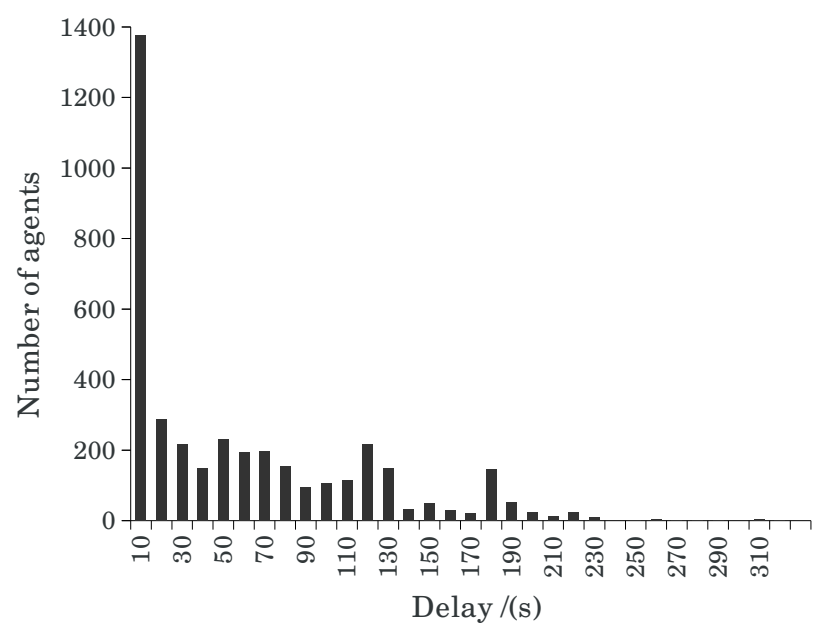

Fig. 7: Time history of cumulative number of agents exited.

possibility of taking unrealistic paths. Detailed comparisons indicate that none of the visitor agents have taken unrealistic path, though only a few has taken the optimal paths. Fig. 8 shows the optimal route of randomly selected non-visitor agents and the routes they have taken. While those routes are realistic choice even for a human, the time differences between most of the paths are small (see Fig. $7)$.

\subsection{Validation of collision avoidance}

The problem setting for validation was set as follows: A target agent was located in a unidirectional flow scenario with a crowd of a certain density in front of him (see Fig. 9). The maximum speed for this agent was set and several simulations with different crowd densities were performed. The agent was traced along its path $(5 \mathrm{~m}$ width sidewalk, walking a distance of $24 \mathrm{~m}$ ) and its average velocity was recorder.

This scenario was choosen trying to mimic the field conditions of the observations reported by Mori and

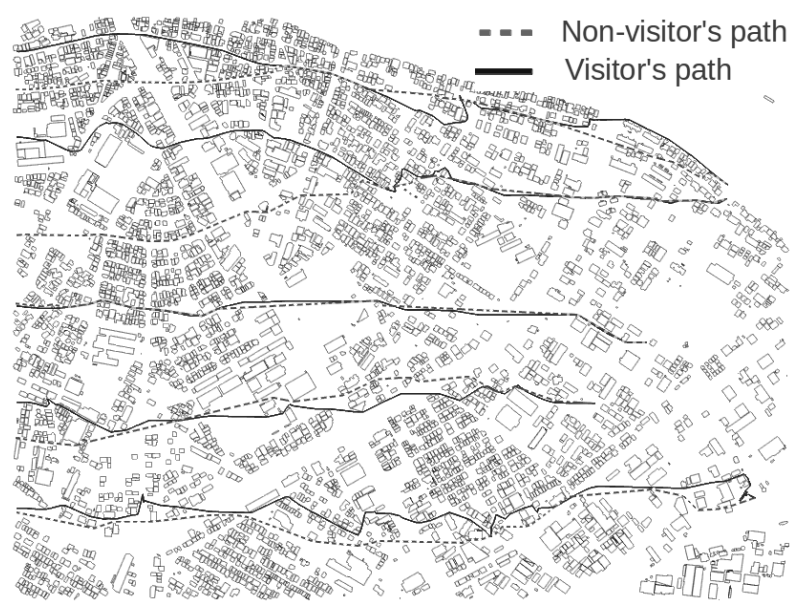

Fig. 8: Comaprison of routes taken by some of the visitor and non-visitor agents with same properties.

Tsukaguchi ${ }^{12)}$. These observations present observations of scenarios with a range of different sidewalks widths, but as can be seen in Fig. 10 our numerical results are in good agreement with their observations. These results shows that the collision avoidance implementation could mimic a specific set of observations. Furthermore to complete the validation we wanted to observe how our numerical results performed in comparison with more general observations, for this purpose we compared our results with the observations of Wiedermann ${ }^{13)}$. Wiederman presents data collected of 25 publications containing field studies and experimental research; it is based on different flow scenarios and boundary conditions. Given the large amount of information it encompasses and the generality of its observations it is commonly used as a benchmark for the evaluation of pedestrian movements in crowds. As can be seen in Fig. 11, our results are again in good agreement with their observations.

The objective of this study is to identify the effectiveness of different strategies for smoothening evacuation process and reducing evacuation time. Strategies giving rise to dense crowds are not an acceptable solution for this emergency situation. Hence we did not check the validity of our adopted ORCA implementation for densities higher than 2.5 people $/ \mathrm{m}^{2}$.

\section{Parallel computing enhancements}

Evacuation simulation with millions of people in large urban areas requires efficient utilization of parallel computing resources. The existing MAS based pedestrian simulation studies have reported low scalability which is limited to a few tens of CPU cores. With several strategies for hiding and minimizing communications, we attained near linear scalability at least up to a few thousands of CPU cores, with several millions of agents ${ }^{7)}$. The rest of this section provides a brief description of some difficulties in parallelization and main strategies used to attain high parallel scalability. 


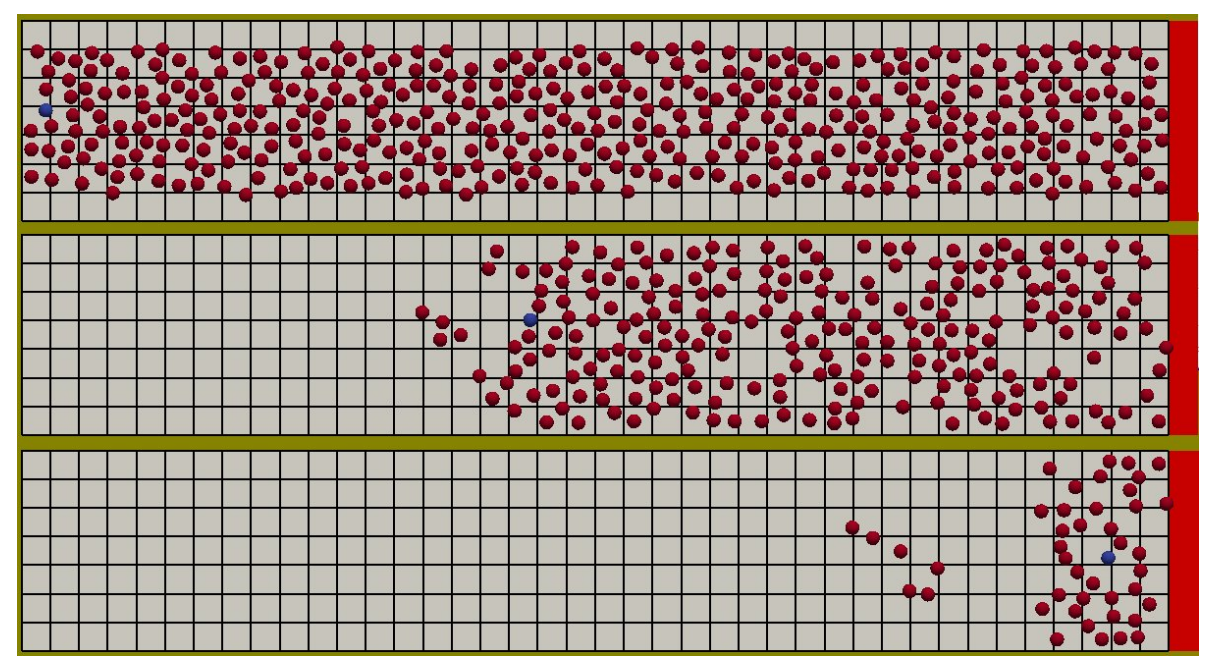

Fig. 9: Agent distribution at three different stages of one of the considered scenarios. Shown in blue is the target agent.

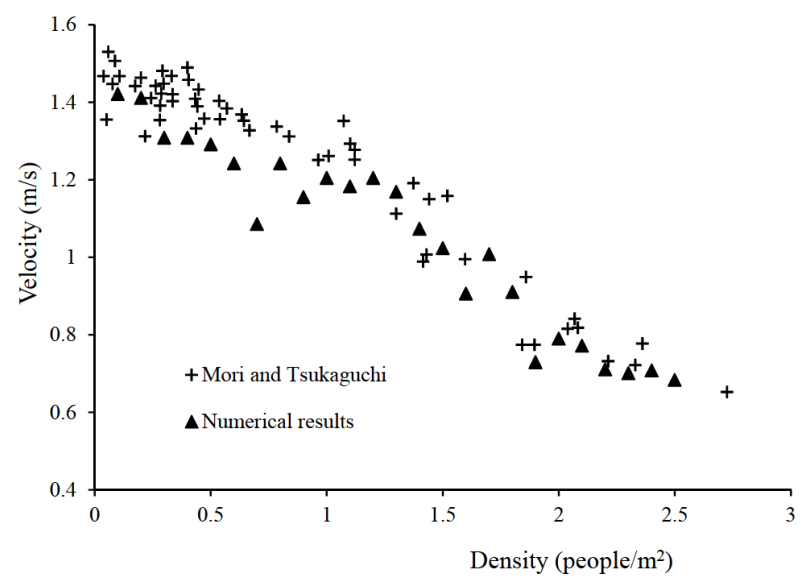

Fig. 10: Comparison of simulation results with field observation reported by Mori and Tsukaguchi. Target agent maximum speed $1.5 \mathrm{~m} / \mathrm{s}$.

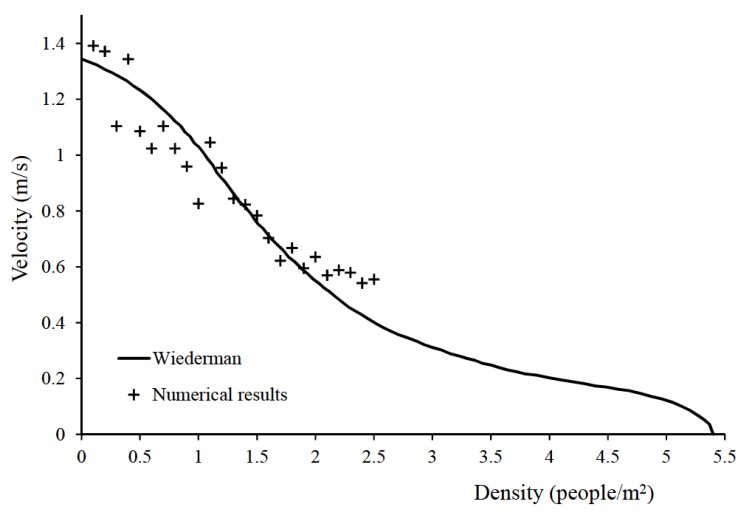

Fig. 11: Comparison of simulation results with field observation reported by Weidmann. Target agent maximum speed is $1.4 \mathrm{~m} / \mathrm{s}$.

The major steps in parallelizing the multi agent code are the same as in other particle type simulations, like SPH. However, compared to other particle type methods, par- allelization of the multi agent code involves several additional difficulties, some of which are listed below.

1. Agents involve large amount of data some of which are dynamically growing and rquire complex data structures like graph.

2. Objects of different types of agents have to be stored in non-contiguous locations.

3. Require maintaining a wide ghost layer of thickness at least equal to the maximum visibility distance of an agent. In a dense urban area, ghost layer of $50 \mathrm{~m}$ may contain a large number of people.

4. Amount of computations depends on the type and surrounding conditions of an agent.

\subsection{Strategies for enhancing scalability}

As mentioned, the basic parallelization strategies are the same as in other particle type simulations; the domain is decomposed such the each has equal work loads, ghost or overlapping layer is maintained and updated with the neighboring CPUs to preserve the continuity and domain is repartitioned when the agent movements bring significant load imbalance. For domain decomposition, kd-tree is used since its simple geometry makes it easy to manage movement of agents between CPUs. In addition to these common strategies, the following major strategies are used to deal with some of above mentioned additional difficulties.

\section{(1) Hiding communications and minimizing volume of data exchanged}

The main strategy of gaining scalability is hiding the communication overhead by first processing the agents to be communicated and posting the message. However, the presence of dynamically growing data makes it difficult to eliminate the communication overhead completely. Since exchanging all the dynamic data requires at least two messages and packing and unpacking of large amount of data, only the new contents added at a time step are exchanged when updating ghost boundaries. This, still, re- 
quires explicit packing and unpacking making it impossible to hide the communication overhead completely. However, a significant portion of communication overhead is hidden, making the code to attain high parallel scalability.

\section{(2) Minimizing data exchange in repartitioning}

Repartitioning for dynamic load balancing is an expensive step since agents have a large amount of data. With 2D-tree partitioning, most of the agents remain in the same CPU even after repartitioning, unless MPI_Dist_graph_create() maps a partition to a different CPU. The repartitioning algorithm detects whether a partition is assigned to the same CPU and exchange only the newly assign agents. This drastically reduces the communication overhead involved with repartitioning, effectively lowering any performance degeneration due to repartitioning.

\subsection{Scalability}

Preliminary scalability tests in the K-computer, Kobe, produced $94 \%$ strong scalability with 2048 CPU cores; strong scalability is defined as $\frac{\left(\frac{T_{m}}{T_{n}}\right)}{\left(\frac{n}{m}\right)}$, where $T_{k}$ is the time with $k$ number of CPU cores and $n \geq 2 m$. A prblem with 2 million agents in $18 \mathrm{~km}^{2}$ urban area is considered in these tests. Figs. $12 \mathrm{a}$ and $12 \mathrm{~b}$ shows the runtime for 400 iterations, excluding and including the repartitioning time. Fig. 12a clearly show the significant performance gain due to repartitioning. As is seen in Fig. 12b the major bottleneck in the current code is repartitioning, which is still a serial code.

\section{Concluding remarks}

We implemented radar vision based autonomous navigation algorithms and adopted optimal reciprocal collision avoidance algorithm (ORCA) ${ }^{6}$ for modelling collision avoidance. The verification test on the autonomous navigation algorithms shows that the proposed navigation algorithms enable both the visitor and non-visitor agents to navigate successfully through urban environments. Although non-visitor agents do not take the optimal paths, their paths are found to be acceptable choices even for human counterparts. Though further improvements to the navigation algorithms are possible, the moderately complex current algorithms are sufficient for the long term objective of real-time observation, prediction and guiding system.

When adopting ORCA algorithm for collision avoidance, we added more ORCA planes to make sure that an agent moves towards the path selected by the navigation algorithm and ordered them in order of importance. This approach allows us to easily relax the conditions of the linear program in case an optimal solution isn't found with the original conditions. Also, aceleration control is included to filter out imposible human movements. The adopted ORCA algorithm implementation is validated by comparing with two field observations and it is found to

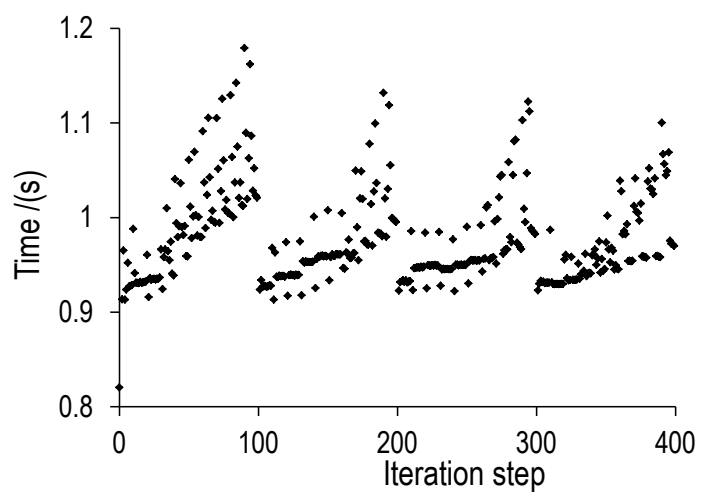

(a) excluding the repartitioning time

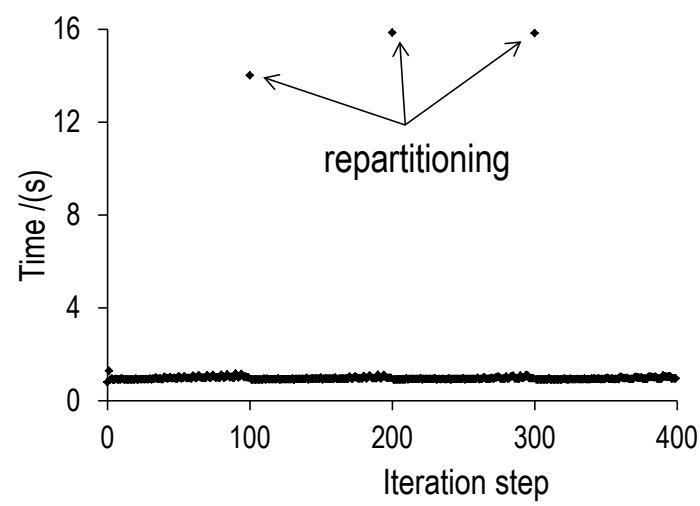

(b) including the repartitioning time

Fig. 12: History of run time with 2048 CPU cores.

be in good agreement with the both, in the required range of crowd densities for the target problem. In order to meet the heavy computational demands of simulating millions of agents, we implemented a scalable MPI based parallel extension; near linear scalability is attained up to 2048 $\mathrm{CPU}$ cores in the $\mathrm{K}$ computer.

\section{Acknowledgements}

This work was supported by JSPS KAKENHI Grant Number 24760359. Part of the results is obtained by using the $\mathrm{K}$ computer at the RIKEN Advanced Institute for Computational Science.

\section{REFERENCES}

1) S. Gwynne, E. Galea, M. Owen, J. Lawrence, and L. Filippiddis, A Review of the MethodologiesUsed in Evacuation Modeling. Fire and Materials, 23, pp. 383-388, 1999.

2) D. Helbing, I. J. Farkas, P. Moln $\tilde{A}_{j}$, T. Vicsek, Simulation of pedestrian crowds in normal and evacuation situations, Pedestrian and Evacuation Dynamics, Springer, pp. 21-58, 2002.

3) M. Hori, H. Miyajima, Y. Inukai and K. Oguni, Agent simulation for prediction of post-earthquake mass evacuation, Journal of Japan Society of Civil Engi- 
neers, Ser. A, vol. 64, pp. 1017-1036, 2008.

4) R. Dulam, M. Lalith, M. Hori, T. Ichimura and S. Tanaka, Development of HPC enhanced multi agent simulation code for tsunami evacuation, Journal of Applied Mechanics, JSCE, vol. 15, 2012.

5) Takashi Saito and Hiroshi Kagami, Simulation of evacuation behavior from tsunami untilizing multi agent simulation, Proceedings ot the 13-th World Conf. on earthquake Engineering, Vancouver, 2004.

6) Jur van den Berg, Stephen J. Guy, Ming Lin, and Dinesh Manocha Cédric Pradalier, Roland Siegwart, and Gerhard Hirzinger, Reciprocal n-body collision avoidance , Robotics Research: The 14th International Symposium ISRR, Springer Tracts in Advanced Robotics, vol. 70, Springer-Verlag, pp. 3-19, 2011.

7) Maddegedara Lalith, Hori Muneo and Ichimura Tsuyoshi, Parallel scalability enhancements of seismic response and evacuation simulations of IES, Proceedings of the 10th International Meeting on HighPerformance Computing for Computational Science, Kobe 2012.

8) Beeson, Jong, and Kuipers, Towards autonomous topological place detection using the Extended Voronoi Graph. IEEE International Conference on Robotics and Automation (ICRA-05), pp. 4373 4379, 2005.
9) Helbing, D., and P. Molnár. Social force model for pedestrian dynamics. Physical Review E51:4282-7, 1995.

10) Jur van den Berg, Ming C. Lin, Dinesh Manocha, Reciprocal Velocity Obstacles for Real-Time MultiAgent Navigation, Proceedings of the IEEE International Conference on Robotics and Automation (ICRA), 2008.

11) A. Nash, K. Daniel, S. Koenig and A. Felner, Theta*: any angle path planning on grids, Proceedings of the AAAI Conference on Artificial Intelligence, pp. 1177-1183, 2007.

12) M. Mori and H. Tsukaguchi, A new Method for Evaluation of Level of Service in Pedestrian Facilities, Transp. Res.-A Vol. 21 A, No. 3, pp. 223-234, 1987.

13) U. Weidmann, Transporttechnik der Fussgänger, Transporttechnische Eigenschaften des Fussgängerverkehrs (Literturauswertung), Schriftenreihe des IVT Nr. 90, Zweite, ergänzte Auflage, Zürich, 1993.

(Received March 18, 2013) 